\title{
APORTES METODOLÓGICOS EN LA INVESTIGACIÓN
}

Rebeca E. Tapia Carlín *

\section{RESUMEN}

La investigación en el ámbito educativo mexicano ha cobrado fuerza en la última década. Las instituciones de educación superior (IES) se han convertido en las principales responsables frente a la sociedad de señalar caminos y proveer, tanto a mujeres como a hombres, de estrategias para encontrar información y procesarla para convertirla en conocimiento (Montaño, 200 , p. 5). De hecho, los procesos de profesionalización tanto de educadores como de formadores docentes se están viendo reflejados en los productos que generan, a saber, artículos de investigación, tesis de grado y documentos recepcionales. El presente artículo basado en la conferencia impartida en el Seminario de Investigación 2007 en la Universidad la Salle- Benavente (ULSA-B) tiene como objeto ayudar al lector a reflexionar acerca de los elementos metodológicos necesarios para llevar a cabo investigación, relacionados con tesis de maestría y documentos recepcionales de las licenciaturas y postgrados de esta institución. Para lograr este objetivo, el texto contiene seis preguntas con sus respuestas. Cabe mencionar que estas preguntas surgieron de un sondeo realizado a los docentes asesores de documentos recepcionales de la ULSA-B a nivel licenciatura, principalmente. En dicho sondeo, los docentes externaron sus principales preocupaciones al asesorar a los alumnos en el desarrollo de su documento recepcional. Sin embargo, la conferencia dictada sobre el tema y el presente artículo pretenden beneficiar a docentes y alumnos de nivel licenciatura y maestría de la ULSA-B, así como, a alumnos de licenciaturas y maestrías en educación que se encuentren realizando investigación.

\begin{abstract}
Investigation in the Mexican educative scope has acquired strength in the last decade. The Higher Education Institutions (IES) have become the main people in charge for society to indicate ways and to provide, as much to women as to men, of strategies to find information and process it to turn it into knowledge (Montaño, 2001, P. 5). In fact, the processes of professionalization as much of educators as of educational trainers are being reflected in the products they generate, that is to say, articles of investigation, theses of bachelor's degree and degree documents. The present article based on the conference distributed in the Seminary of Investigation 2007 at La Salle University- Benavente (ULSA-B) takes as main objective to help the reader reflect about the methodological elements necessary to carry out investigation, related to thesis of masters and degree documents of the bachelor's and postgraduate programs of this Institution. In order to achieve this objective, the
\end{abstract}


text contains six questions with their answers. It is important to add that these questions arose from a questionnaire mainly ap- plied to educational advisers of reception documents of the ULSA-B at bachelor's degree level. In this questionnaire, advisers expressed their main concerns when assessing students in the development of their degree document. Nevertheless, the conference dictated on the topic and the present article try to benefit teach- ers and students from bacherlor's and master's degree from the ULSA-B, as well as, students of bachelor's and master's degree in Education who are currently doing research.

* Rebeca E. Tapia Carlín Doctora en Lingüística Aplicada, Maestra en la Enseñanza del Inglés y licenciada en Educación. Es catedrática de Posgrado en la ULSA Benavente y articulista y Miembro del Comité Editorial de la Revista de la Asociación Mexicana de Maestros en Inglés.

\section{INTRODUCCIÓN}

La primera pregunta inicia indagando la definición, semejanzas y diferencias entre un documento recepcional y una tesis en la ULSA-B. Tanto el documento recepcional como la tesis son reportes de investigación; el documento recepcional se redacta a nivel licenciatura y la tesis a nivel maestría. Las diferencias estriban en el tipo de investigación que re- quiere cada uno y el tono en que se redactan. El documento recepcional se realiza con investigación de tipo cualitativa y por lo mismo su tono de redacción es más de tipo personal. Por otro lado en las tesis de maestría en la ULSA-B se realiza principalmente investigación cuantitativa y en menor grado investigación cualitativa, lo que conlleva un tono de redacción impersonal que es el tipo de redacción empleado frecuentemente en artículos de investigación a nivel internacional (Swales, 990).

La segunda pregunta se refiere a las diferencias entre investigación cuantitativa y cualitativa en ciencias sociales, especialmente en el área educativa. La investigación cuantitativa analiza la realidad educativa a través del análisis de información numérica (estadística) para probar hipótesis. Es inflexible y objetiva. Estudia lo explícito y visible de los fenómenos. Por otro lado, la investigación cualitativa analiza la realidad educativa a través de análisis de información no numérica, para responder preguntas de investigación o para validar o rechazar supuestos. Es flexible y subjetiva; permite hacer visible lo invisible (Calvo, 2007; Hernández, Fernández y Baptista, 2006; Schmelkes, 998; Silverman,2000). Para complementar esta información, se describe la diferencia entre hipótesis y supuestos. "La hipótesis es una respuesta tentativa al problema de investigación. Consiste en una aseveración que puede validarse estadísticamente" (Schmelkes, 998, p. 38). 
Los supuestos también son soluciones tentativas al problema de investigación. Su validez se comprueba mediante información empírica, reglas de lógica o en forma cualitativa (Schmelkes, 998, p. 37). Así, se entiende que las hipótesis y los supuestos son similares, pero su método de validación es diferente. La investigación cuantitativa y cualitativa se complementan ya que cada una nos permite descubrir parte de la realidad.

La tercera pregunta se refiere a las líneas de investigación en la ULSA-B. La respuesta a esta pregunta se obtuvo del Plan Rector de Investigación 2007-20 2 de la institución. A continuación se presentan las mismas.

* Enseñanza de la lectura y la escritura

* Pensamiento lógico-matemático

* Estrategias de enseñanza-aprendizaje

* Inteligencia y/o creatividad

* Inclusión social

* Educación intercultural

${ }^{*}$ Actitudes y valores

* Psicopedagogía clínica

* Asesoramiento psicopedagógico

* Investigaciones teológicas aplicadas (ULSA-B, 2007).

La cuarta pregunta se refiere a los métodos cualitativos para realizar investigación de acuerdo a Álvarez-Gayou (2003). Los métodos que se presentan son ocho.

\section{Observación y auto-observación.}

La observación ha sido muy importante en la generación del conocimiento desde que se tiene noción de la existencia del ser humano. Desde el paradigma cuantitativo existen dos tipos de observación: la del participante y la del no participante. La observación en investigación cuantitativa se realiza con apoyo de listas de cotejo o formatos con categorías pre-diseñadas. En investigación 
cualitativa el proceso puede utilizar formatos en donde las preguntas son abiertas. Asimismo, las notas de campo le sirven al investigador cualitativo para rescatar los elementos descubiertos a través de la observación de tipo émica o etnográfica. La observación es indispensable en procesos de investigación cualitativa (Hernández et al, 2006. p. 597). En la investigación cualitativa sólo se considera que la observación puede ser participante, ya que se asume que el investigador no se puede separar por completo del fenómeno estudiado (Álvarez-Gayou, 2003, pp. 03- 04). Silverman (2000, p. 89) afirma que la observación es fundamental para entender otra cultura y por ende el fenómeno estudiado.

La auto-observación consiste en que el investigador se estudia a sí mismo y a sus colegas; este tipo de observación es ampliamente reconocido en la investigación cualitativa (Álvarez-Gayou, 2003, p. 08) y se puede realizar a través de audio o video grabaciones de lecciones y diarios de enseñanza y/o de aprendizaje (Richards, 990 en Bailey, Curtis y Nunan, 200z , p. 27).

\section{Entrevista y Cuestionario.}

Una entrevista es una conversación que tiene una estructura y un propósito. En la investigación cualitativa, la entrevista busca entender el mundo desde la perspectiva del entrevistado y desmenuzar los significados de sus experiencias (Álvarez-Gayou 2003, p. 09). En la investigación cualitativa las entrevistas y los cuestionarios contienen respuestas predeterminadas y se aplican a muestras representativas de la población elegidas al azar.

En la investigación cualitativa las preguntas de entrevistas y cuestionarios son abiertas o semiestructuradas y se aplican a muestras pequeñas a menudo elegidas a propósito para que los sujetos sean los que poseen ciertas características o cualidades (Silverman, 2000).

\section{Fotobiografía y autobiografía.}

Ambas técnicas cualitativas le piden al sujeto que narre su vida. La fotobiografía emplea fotografías de familia que le permiten crear al participante historias personales (Álvarez-Gayou, 2003, pp. 3-4). Por otro lado, la autobiografía es una técnica narrativa que produce un texto retrospectivo de la vida del individuo. Hernández et al (2006) le llaman biografía o historia de vida (p. 6 9). Se le asocia también a la historia oral (Álvarez-Gayou, 2003, pp. 26- 27) en la que se puede entrevistar a más de un sujeto para complementar la información acerca de la vida de una persona.

\section{Análisis narrativo.}

El análisis narrativo es una técnica en la cual se recopilan historias y el objeto a 
investigar es la historia misma (Álvarez-Gayou, 2003, p. 26). Las narraciones pueden comprender autobiografías, narraciones de hechos, diarios de aprendizaje/enseñanza e interactivos, memorias y diarios personales. Hernández et al (2006, pp. 70 -706) explican como llevar a cabo un estudio narrativo, ya sea para presentar la solución de un problema o para poder entender un fenómeno.

\section{Grupo focal o de enfoque.}

Esta técnica es una especie de entrevista grupal en la que los partici- pantes se reúnen en grupos de 3 a 0 personas y conversan en torno a uno o varios temas en un ambiente relajado e informal bajo la con- ducción de un investigador o idealmente un especialista en dinámicas grupales (Hernández et al, 2006, p. 605).

\section{Investigación endógena.}

Esta investigación se genera desde los propios miembros del grupo aunque pueden estar asesorados por un investigador externo. Los participantes eligen un objetivo, el foco de interés, la metodología y el diseño de la investigación (Álvarez-Gayou, 2003, p. 46).

\section{Investigación acción.}

El propósito de la investigación acción es resolver problemas cotidianos e inmediatos (Álvarez-Gayou, 2003, p. 59). Esta técnica emplea los siguientes pasos de acuerdo a Bailey (et al. p. 37).

* Detección del problema

* Investigación preliminar

* Reflexión y desarrollo de la hipótesis

* Plan de intervención y acción

* Monitoreo y recolección de información

* Observación del resultado

* Regreso a la reflexión y continuación del ciclo.

\section{Análisis del contenido.}

Esta técnica desde el punto de vista cualitativo es una manera de análisis de cualquier forma de comunicación humana, especialmente la emitida por los medios masivos de comunicación y por personajes populares. Los pasos que se sugieren para llevar a cabo esta técnica son:

* Determinar qué contenido se estudiará y por qué

* Determinar qué elementos se van a buscar

* Definir el campo de observación del contenido

* Definir la forma de recabar información de acuerdo al propósito de la investigación

* Unificar criterios para observación y codificación si son varios los codificadores (Álvarez-Gayou, 2003, p. 59). 
La quinta pregunta aborda las técnicas necesarias para el análisis e interpretación de resultados. Las recomendaciones que Hernández et al (2006) y Schmelkes ( 998) hacen al respecto se han resumido en tres enunciados:

- Retomar los objetivos, las preguntas de investigación y los supuestos y/o la hipótesis de trabajo. En el documento recepcional se recomienda remitirse al cuadro de concentración, en la tesis se recomienda retomar el capítulo .

- Leer detenidamente la información obtenida a través de los instrumentos varias veces y organizar los resultados en tablas cualitativas (codificación) o estadísticas (gráficas) presentando así la solución al problema o dando respuesta a las preguntas; validando los supuestos y/o probando o rechazando la hipótesis.

- Siempre que sea posible es altamente recomendable triangular la in-formación para dar validez al estudio.

La sexta pregunta se refiere a recomendaciones generales para llevar a cabo investigación en ciencias sociales de una manera más eficiente. En este tema se presentan cuatro sugerencias.

-Documentar la investigación con investigaciones realizadas anterior- mente (Hernández et al, 2006, p. 53 ).

- Llevar un diario de investigación para anotar el proceso con detalle y poder recuperar información importante (Schmelkes, 998, p. ).

- Documentar el tema lo más posible; sistematizar el uso de referencias bibliográficas aplicando el formato APA y llevando un registro de las mismas para poder incluirlas tanto en el texto como en la lista de referencias bibliográficas que se anota al final del trabajo (Ibáñez, 995).

- Elegir un problema de investigación que nos interese y que estemos suficientemente interesados en él para dedicarnos sin perder el interés en el mismo.

- Trabajar con entusiasmo y dedicación; organizar nuestro tiempo y no detenernos sino hasta terminar.

\section{CONCLUSIONES}

Espero que estos aportes y reflexiones a la metodología de la investigación para documentos recepcionales y tesis sean de utilidad tanto a docentes como alumnos. Concluyo deseándoles mucho éxito en las investigaciones que realicen en el ámbito educativo, especialmente para documentos que son necesarios para la obtención del grado. Es importante señalar que dichas investigaciones pueden lograr que la educación en México vaya mejorando gracias al esfuerzo de muchos profesionales de la educación como tú o yo. 
FUENTES DE CONSULTA:

- ALVAREZ-gayou, J. L. (2003). Cómo hacer investigación cualitativa: Fundamentos y metodología. México, D.F: Paidós.

- BAILEY, K.M., Curtis, A. y Nunan, D. (200 ). Pursuing professional development: The self as a source. Boston, MA. : Heinle \& Heinle.

- CALVO, B. (2007). La perspectiva histórico-cotidiana en la investi gación etnográfica en la educación. Manuscrito presentado en la 5ta Jor- nada de Investigación Educativa organizada por la Universidad la Salle- Benavente en la ciudad de Puebla, Pue. el 22 de marzo del 2007.

- HERNÁNDEZ, R.; Fernández, C. y Collado, P. (2006). Metodología de la investigación. México, D.F.: Mc Graw-Hill.

- IBÁÑNEZ, B. ( 995). Manual para la elaboración de tesis. México, D.F. : Trillas.

- MONTAÑO, P. (200 ). Metodología de la investigación educativa :Sin- tagma.

- SCHMELKES, C. ( 998). Manual para la presentación de anteproyec- tos e informes de investigación (tesis) . México, D.F.: Oxford University Press.

- SILVERMAN, D. (2000). Doing qualitative research: A practical hand-book. London: Sage.

- SWALES, J.M. ( 990). Genre analysis: English in academic and research settings. Cambridge: Cambridge University Press.

Universidad la Salle-Benavente. (2007). Plan rector de investigación 2007-2012. Puebla: ULSA-B. 